livraisons

d'Histoire

de l'Architecture

\section{Livraisons de l'histoire de l'architecture}

$31 \mid 2016$

La source photographique dans la pratique de I'historien de l'architecture

\title{
L'Observatoire photographique national du paysage : archive rétrospective et prospective des territoires
}

The National photographic landscape observatory: retrospective archive and territories' foresight

Das Observatoire photographique national du paysage - ein retrospektives wie prospektives Archiv der Landschaft

\section{Frédérique Mocquet}

\section{OpenEdition}

Journals

Édition électronique

URL : http://journals.openedition.org/lha/589

DOI : 10.4000//ha.589

ISSN : 1960-5994

\section{Éditeur}

Association Livraisons d'histoire de l'architecture - LHA

Édition imprimée

Date de publication : 14 juillet 2016

Pagination : 51-64

ISSN : 1627-4970

Référence électronique

Frédérique Mocquet, «L'Observatoire photographique national du paysage : archive rétrospective et prospective des territoires », Livraisons de l'histoire de l'architecture [En ligne], 31 | 2016, mis en ligne le 14 juillet 2018, consulté le 21 avril 2019. URL : http://journals.openedition.org/lha/589 ; DOI : 10.4000/ Iha.589

Ce document a été généré automatiquement le 21 avril 2019

Tous droits réservés à l'Association LHA 


\title{
L'Observatoire photographique national du paysage : archive rétrospective et prospective des territoires
}

\author{
The National photographic landscape observatory: retrospective archive and \\ territories' foresight \\ Das Observatoire photographique national du paysage - ein retrospektives \\ wie prospektives Archiv der Landschaft
}

Frédérique Mocquet

\section{Architecture, territoire, photographie et institution : un lien originel}

1 Un lien ancien unit architecture, territoire, photographie et institution. En 1851, la commission des monuments historiques lance une commande pour « recueillir des dessins photographiques d'un certain nombre d'édifices historiques ${ }^{1}{ }^{\prime}$, envisageant alors l'outil photographique comme moyen de relevé exact et exhaustif du patrimoine architectural français. La Mission héliographique ${ }^{2}$, qui réunit cinq photographes membres de la Société héliographique, est la première commande publique collective de l'histoire de la photographie. Elle affirme une reconnaissance officielle du procédé, scelle le lien avec l'institution et jette les bases d'une pratique alors nouvelle: la vue d'architecture.

2 C'est à une autre échelle et à une autre dimension que celle de l'inventaire visuel du bâti que s'intéresse l'Observatoire photographique national du paysage (OPNP). Depuis la fin des années 1980, en France, des commandes publiques pensent la photographie comme un outil d'identification, de connaissance et d'action selon trois préoccupations situant les travaux entre étude technique et enquête culturelle et sensible : état des lieux, état 
des perceptions, état des évolutions du paysage. Participant de cette dynamique de projets associant photographie et territoire, l'OPNP porte un intérêt privilégié, à travers la question de l'évolution des paysages, aux rapports entre l'espace et le temps.

\section{Le paysage comme catalyseur}

3 Ce programme du ministère de l'Environnement résulte de la combinaison de plusieurs dynamiques $^{3}$ : une attention nouvelle pour la question de l'environnement de la part des institutions, une réflexion culturelle pluridisciplinaire sur celle du paysage et, enfin, un mouvement de reconnaissance de la photographie, qui marque le milieu culturel des années 1980. En France, l'intérêt des pouvoirs publics pour le paysage aboutit, en 1993, à la loi dite «loi paysage ${ }^{4}$ » et, à l'échelle européenne, à la Convention Européenne du Paysage qui vise à mieux le définir et le protéger ${ }^{5}$. Cette prise en compte inédite du paysage est amenée par la dynamique théorique et critique développée dans des champs multiples ${ }^{6}$ : philosophie, esthétique, histoire de l'art, géographie, etc. Le paysage est désigné dans la Convention comme "une partie de territoire telle que perçue par les populations, dont le caractère résulte de l'action de facteurs naturels et/ou humains et de leurs interrelations ${ }^{7}$ » et est pensé comme imbrication d'éléments combinant données culturelles, écologiques, environnementales et sociales. Le paysage des espaces ordinaires et quotidiens, comme ceux des sites exceptionnels, est reconnu " patrimoine culturel et naturel de l'Europe » et acteur de la «qualité de vie» et du «bien être individuel et social ». Il est désormais envisagé comme un bien commun en constante évolution car résultant des interrelations entre actions humaines et facteurs naturels, qu'il convient de surveiller et protéger. Devenu vecteur démocratique, le paysage cristallise des problématiques, enjeux et valeurs relevant de contextes disciplinaires et points de vue différents. Il fait consensus en tant qu'outil de réflexion et d'action, sans pour autant qu'une définition claire et univoque ne soit déterminée.

Concept géographique, écologique et philosophique, levier d'action pour l'aménagement, le paysage en tant qu'objet et forme de représentation relève aussi de la sphère artistique. Il se présente comme une voie par laquelle la photographie est peu à peu reconnue comme une pratique culturelle. En effet, au tournant des années 1980, le genre du paysage trouve une vitalité nouvelle en photographie. En outre, il diversifie un milieu dominé jusqu'alors par le photoreportage et la photographie humaniste. Le paysage contribue à faire entrer le médium dans un processus d'intégration institutionnelle. Mais l'identité floue de la photographie (est-elle un art ou une technique ?) mène à une institutionnalisation chaotique, reflétée par les discours et débats qui agitent un milieu hésitant entre la valorisation artistique et la reconnaissance culturelle du médium et les décisions étatiques. Le ministère de la Culture et de la Communication met en place une ossature complexe (le Patrimoine photographique en 1978, le Centre national de la photographie et l'École supérieure de la photographie d'Arles en 1982), matérialisant une action généraliste qui se diffuse dans les régions et départements (avec par exemple des Centres régionaux pour la photographie). Elle se manifeste également par la multiplication de commandes institutionnelles consacrées notamment au paysage, matérialisant la concordance entre politique culturelle et politique spatiale en mutation. Ainsi, la Mission photographique de la DATAR lancée en $1984^{8}$, devenue aujourd'hui emblématique, est largement financée par le ministère de la Culture 9 . 


\section{De la prise de conscience au projet}

5 C'est dans ce contexte que s'élabore le projet d'un programme photographique consacré à l'observation des paysages et de leur évolution. L'idée émerge dans l'esprit de Jean Cabanel, chef de la Mission du paysage du ministère de l'Environnement ${ }^{10}$ et de Caroline Mollie-Stefulesco, paysagiste chargée de mission. Celle-ci se souvient d'une «forte prise de conscience chez les professionnels de l'anarchie totale générée par les Trente Glorieuses et des retombées de l'agriculture industrielle ${ }^{11}$ » à laquelle doit répondre une sortie progressive de la "politique d'aménagement planificatrice et verticale ${ }^{12}$ » qui prendrait en compte le paysage.

6 Parallèlement à ces constats de terrain, la paysagiste évoque la découverte des images de la Mission photographique de la DATAR comme un « choc émotionnel ». Menée en 1984 et 1989, la commande demande à 29 photographes de représenter le paysage français des années $1980^{13}$. De la réunion de ces projets photographiques se dégage un état des lieux des politiques d'aménagement des Trente Glorieuses, des visions des paysages ordinaires français et une réactualisation du genre de la photographie de paysage. Parfaite synthèse, donc, des problématiques spatiales et culturelles de la période, cette mission fait le lien entre les différents champs et oriente la conception du programme. Le «choc émotionnel » ressenti par Caroline Mollie-Stefulesco a deux causes : d'abord, les travaux de la DATAR mettent en images les conséquences de la politique d'aménagement menée depuis l'après-guerre et des mutations de l'agriculture; ensuite, ce corpus réactualise une culture du paysage par la photographie et provoque à l'échelle individuelle et collective, une « révélation esthétique ».

7 Les deux acteurs de la Mission du paysage estiment qu'il manque des repères pour mesurer les effets et responsabilités publiques des phénomènes complexes induisant la transformation des paysages. Il leur semble, en effet, nécessaire de compléter les outils d'observation par un regard qualitatif : la photographie, médium technique et artistique, d'archive et de création, apparaît comme un moyen idéal permettant de combiner objectifs techniques, culturels et esthétiques. C'est un questionnement pour la quatrième dimension du paysage, celle du temps, qui porte et guide la réflexion. Revenant sur les origines de l'observatoire photographique, la paysagiste évoque une inquiétude face au constat que les temps des espaces et des paysages, courts comme longs, sont à ce moment-là peu pris en compte par les architectes, urbanistes, paysagistes. Elle souhaite encourager les praticiens à prendre en compte ce facteur au sein de leurs interventions. Le temps doit, dans sa complexité, dans sa dimension prévisible comme non-prévisible, être envisagé comme un enjeu de territoire et de paysage. Comme on oublie très vite ce qui a été, que la mémoire est sélective et stratifiée et que le « voir » agit différemment du "dire », on fait appel à la force d'évidence de la photographie et on convoque l'archive; affirmant alors une filiation avec l'histoire du médium, marquée par l'association entre institution, aménagement du territoire et photographie.

8 Finalement, l'OPNP est annoncé en Conseil des ministres le 22 novembre 1989. Jean Cabanel le présente comme un programme visant à "constituer un fonds de séries photographiques qui permette d'analyser les mécanismes et les facteurs de transformations des espaces ainsi que les rôles des différents acteurs qui en sont la cause de façon à orienter favorablement l'évolution du paysage.» Le pouvoir central, en validant ce programme, reconnaît les complexités des évolutions des espaces et des 
paysages : évolutions résultant souvent de petits changements difficilement perceptibles isolément et de l'action combinée de différents acteurs, publics et privés, collectifs et individuels. Il admet ainsi la partialité de sa maîtrise par sa politique d'aménagement et, au-delà, reconnaît la diversité des responsabilités, y compris la sienne, dans leurs conséquences. Avec l'OPNP, l'État met en place un instrument d'appréciation des politiques publiques et esquisse la possibilité d'une autocritique (ill. 1) (ill. 2).

III. 1 : Rue F. Debergue (09000501), février 1997



Itinéraire n 9, Montreuil-sous-Bois, créé en 1997.

(c) Cl. Anne Favret et Patrick Manez, Observatoire photographique national du paysage. Photographie originale en couleurs 




Itinéraire n 9, Montreuil-sous-Bois, créé en 1997.

(c) Cl. Anne Favret et Patrick Manez, Observatoire photographique national du paysage. Photographie originale en couleurs

\section{L'archive comme source et modèle : fondements techniques et épistémologiques}

9 Le fonctionnement de ce programme, en tant que dispositif technique proposé aux acteurs locaux des territoires ${ }^{14}$, est conditionné par l'application d'une méthode élaborée par Caroline Mollie-Stefulesco, qui fait appel à une documentaliste, Véronique Ristelhueber, une paysagiste, Anne Velche et ensuite un photographe, Daniel Quesney. Élaborée au fil des expériences de terrain, entre 1992 et 1999, cette méthode est photographique et archivistique, d'une part, et administrative, d'autre part. Elle est en effet un outil de mise en forme et d'encadrement pratique d'une action à la fois nationale et locale sur les territoires, motivé par des ambitions de légitimation politique et symbolique de ses résultats.

10 Les deux fonctions combinées de l'OPNP, l'une rétrospective et l'autre prospective, orientent simultanément le programme vers des corpus existant et vers la création d'un fonds contemporain. Véronique Ristelhueber mène une recherche d'archives et de références photographiques pouvant constituer à la fois des éléments de modèles méthodologiques pour l'observation des territoires et un matériau de base pour la constitution $\mathrm{du}$ fonds contemporain. La Mission du paysage découvre ainsi «la photographie de territoire ", pratique inhérente à l'identité américaine mais moins ancrée dans la culture française. Menée dans la seconde moitié du XIX ${ }^{e}$ siècle sous la 
forme de surveys, c'est-à-dire de missions accompagnant la conquête des grands espaces, ces images de photographes emblématiques, comme Timothy O'Sullivan et William Henry Jackson, sont l'objet du projet du photographe Mark Klett, qui les reproduit à l'identique entre 1977 et 1979 et réactualise ainsi les paysages de sites constituant l'imaginaire des territoires états-uniens ${ }^{15}$. Le même procédé est utilisé par Alain Blondel et Laurent Sully-Jaulmes (respectivement galeriste et photographe), qui travaillent à partir des années 1970 à représenter l'évolution urbanistique de la banlieue parisienne en re-photographiant cartes postales et images d'archives ${ }^{16}$. Ces fonds sont des témoignages de manières de voir, de faire et de représenter le territoire. Plus encore, les images du service de Restauration des Terrains de Montagnes (RTM) mis en place en 1882, d'une qualité technique et esthétique exceptionnelles, sont exemplaires de l'utilisation de la technique photographique par une administration en charge de la gestion d'un territoire ${ }^{17}$.

Le travail de repérage est conséquent et en 1999, près de trois mille séries rétrospectives datant principalement du début $\mathrm{du} \mathrm{XX}^{\mathrm{e}}$ siècle sont identifiées, ayant pour thèmes la ville, la banlieue, les bourgs et villages, la montagne, etc. En sont extraits les principes qui présideront à la méthodologie de l'observatoire: l'usage d'archives (fonds photographique, cartes postales anciennes dont la production est très active au début du $\mathrm{XX}^{\mathrm{e}}$ siècle), la nécessité de produire un ensemble visuel cohérent sur un territoire, l'enjeu d'en montrer les évolutions en respectant les règles de la re-photographie, nommée reconduction. Ce matériau historique, outre sa qualité de modèle méthodologique, propose des matières premières pour d'éventuels re-photographiés du volet prospectif de l'OPNP : ce sont alors les paysages, leur physionomie et les manières de les représenter, qui sont reconduits, et les différences mises en lumière. L'Observatoire, «veille photographique » destinée à porter un regard contemporain et public sur les territoires, travaille inlassablement le lien entre passé, présent et avenir des espaces. En proposant de constituer une archive pour l'avenir, un pari fort est fait, qui est porté par une croyance en l'utilité future de documents produits aujourd'hui. Ces photographies qui proposent une réflexion pour le futur produisent donc simultanément une archive de ce qui a été.

Deux outils conceptuels sont développés par la méthode: d'une part, les séries photographiques et, d'autre part, les itinéraires d'observation. Sur chaque territoire, l'itinéraire d'observation est constitué de cent points de vue. Parmi eux, seule une sélection de quarante sera re-photographiée annuellement, conformément aux conditions de prise de vue initiale : cadrage, focale, heure et saison. Les soixante autres constituent une mémoire et une réserve mobilisable. Le respect des règles de la reconduction est un des fondements méthodologiques découlant du caractère évolutif du paysage et dépendant de l'aptitude de l'objet photographique à offrir une visibilité des complexités des évolutions à l'œuvre non appréhendables in situ. Une série photographique est une succession de prises de vue effectuées depuis le même emplacement selon le même cadrage. Elle est établie à partir d'une capture initiale qui fait l'objet d'une ou plusieurs reprises de vue. Les séries contemporaines sont celles dont l'image première a été réalisée dans le but d'être reconduite et les séries rétrospectives, celles effectuées à partir d'images préexistantes. Un itinéraire d'observation contient donc quarante séries photographiques (les images initiales et leurs reconductions) (ill. 3) (ill. 4) ${ }^{18}$. 
III. 3 : Saint-Paul-et-Valmalle, avant et après, A 75 (02002301), novembre 1992

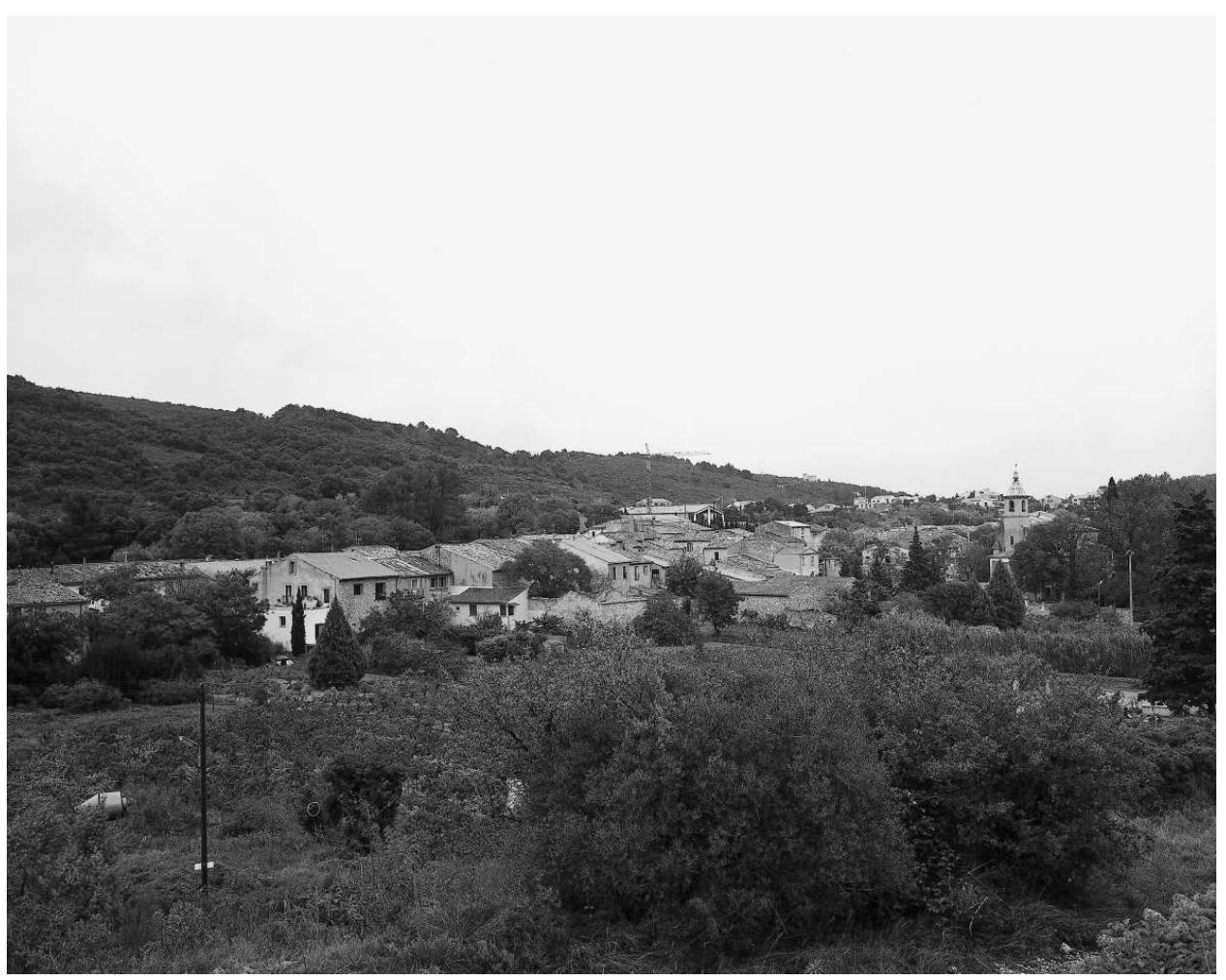

Itinéraire n² 2, département de l'Hérault (CAUE), créé en 1992.

(c) $\mathrm{Cl}$. Raymond Depardon, Observatoire photographique national du paysage 


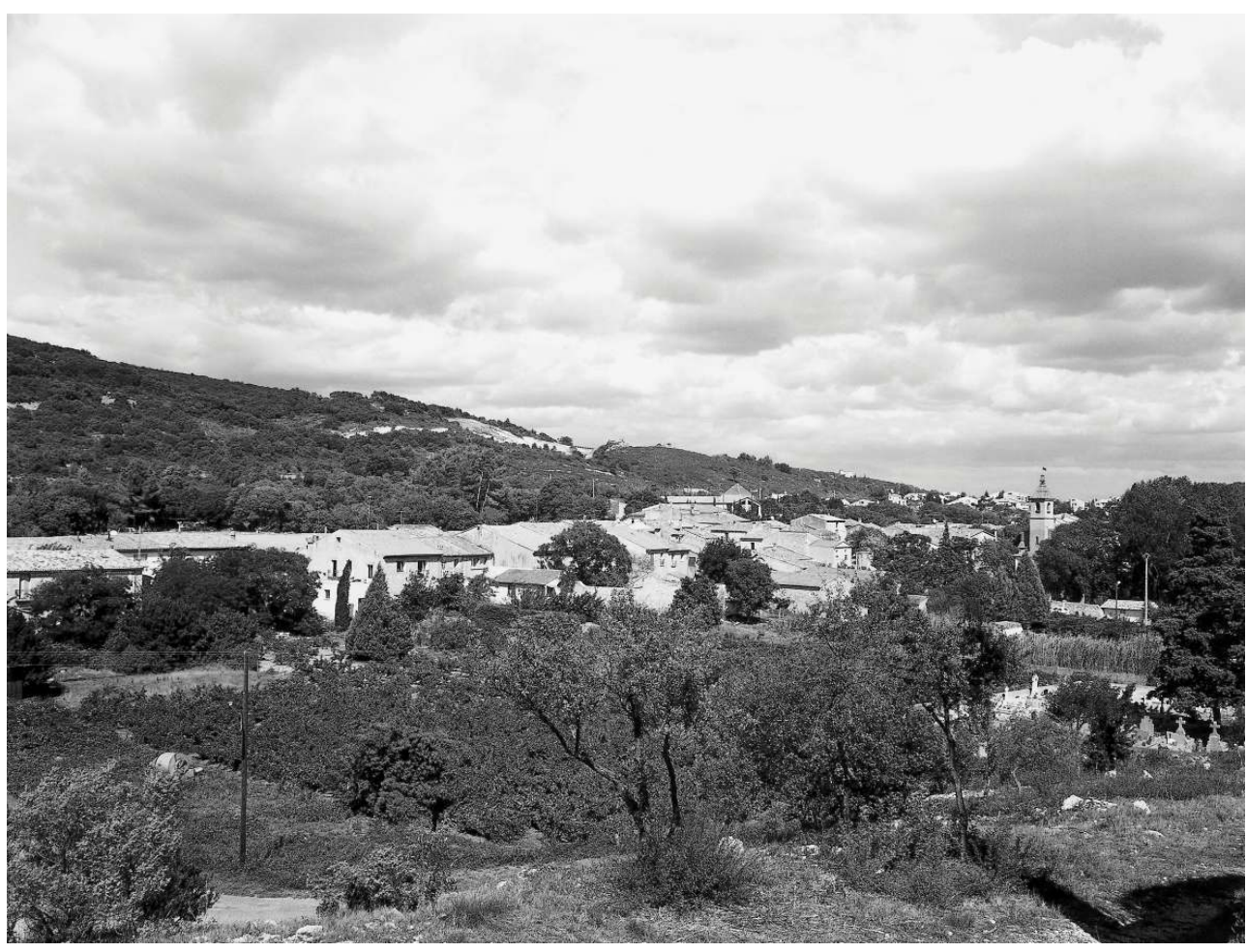

Itinéraire n² 2, l'Hérault (CAUE), créé en 1992.

(c) Cl. Raymond Depardon, Observatoire photographique national du paysage

14 Le préalable, la vertu initiale de chaque itinéraire, est donc un état des lieux montrant la diversité des paysages constitutifs de l'identité d'un territoire. Les règles le définissant expriment les idées fortes portant, selon le ministère de l'Environnement, la notion de paysage. Le nom même d'itinéraire traduit l'importance du déplacement et de la multiplicité des points de vue sur un même territoire pour en « extraire » les paysages. Le procédé de la reconduction met en avant le rôle du temps et la contrainte des prises de vue à hauteur de la vision humaine, celle de la perception de l'individu dans la constitution des paysages. Aussi, la contrainte de produire des images depuis l'espace public exclusivement revendique le caractère commun du paysage et rappelle qu'un paysage ordinaire est aussi un paysage vécu à apprécier au quotidien.

\section{Projet commun, projet public : la forme et la méthode comme infrastructures}

15 Au-delà de la fonction d'inventaire, l'Observatoire est conçu comme moyen pour observer, comprendre et analyser les paysages. Pensé comme une aide à la décision et plus encore à la conception, il est proposé comme outil complémentaire aux moyens d'analyse habituels des acteurs en charge des territoires, tels que les informations cartographiques et statistiques et vues satellites (qui objectivent et quantifient). Outil technique, l'observatoire, en tant que proposition de représentations, a aussi une dimension culturelle affirmée par ses concepteurs, qui voient en lui le moyen de contribuer à la création d'un « regard contemporain sur le paysage » et d'encourager une 
«culture moderne de l'environnement ${ }^{19}$. " Auprès du grand public, il devient objet de débat, moyen de sensibilisation, dispositif pédagogique.

Pour la Mission du paysage, chaque itinéraire est : d'abord, un projet collectif né de la rencontre entre l'administration centrale, ensuite, un idéal de territoire porté par un maître d'ouvrage public local ayant identifié dans le cadre de sa politique d'aménagement les questions qui se posent et les lieux problématiques, et, enfin, la démarche artistique d'un photographe qui propose son regard à travers des points de vue. Ce projet est donc porté par trois entités qui travaillent ensemble pour le paysage. La méthode est envisagée comme condition et moyen de co-production, de conciliation d'une démarche artistique et d'objectifs techniques. Elle agit comme un outil, une garantie, une infrastructure pratique et conceptuelle aux échelles nationale et locale.

L'OPNP se définit donc par cette méthode photographique et archivistique autant que par les dispositifs mis en place corrélativement pour lui donner une existence pratique et symbolique. Un conseil d'experts réunit plusieurs acteurs importants de l'institution culturelle française et est présidé par Jean-Claude Lemagny, alors conservateur du fonds photographique contemporain de la Bibliothèque nationale. Son rôle est d'assurer la qualité photographique des itinéraires notamment par le choix de photographes expérimentés et reconnus. Un comité de pilotage national, spécialisé dans les questions d'aménagement du territoire, valide les orientations générales: présidé par François Letourneux, directeur du Conservatoire du littoral, il rassemble des acteurs d'institutions comme la DATAR, le ministère de l'Équipement et de l'Environnement, etc. Ces deux instances assurent à la Mission du paysage un encadrement technique et, plus encore, une validité symbolique, ainsi qu'un rayonnement et une légitimité dans la sphère artistique et culturelle, de même que dans le secteur de l'aménagement.

Les itinéraires sont impulsés par l'instance nationale, qui propose des collaborations à des institutions locales: Parcs naturels régionaux, communes ou CAUE par exemple. Chaque partenaire local, lié par une convention tripartite avec l'État, d'une part, et un photographe, d'autre part, voit son rôle de créateur et de gestionnaire précisément défini par la méthode. Il doit, d'abord, formuler des objectifs dans un cahier des charges, puis, créer et présider un comité de pilotage, ensuite, archiver les documents divers constituant l'itinéraire et, enfin, assurer partiellement d'abord et complètement ensuite le financement du projet. Structure complémentaire, le comité de pilotage est composé de représentants des services de l'État et d'organismes dédiés aux territoires (CAUE, Bâtiments de France, etc.), des assemblées régionales, départementales, etc., des réseaux professionnels et associatifs. Il est le groupe de travail opérationnel qui accompagne les deux maîtres d'ouvrage (l'État et le partenaire local) pour la formulation dans le cahier des charges des problématiques et des lieux à observer ainsi que des attentes, la récolte de fonds photographiques existants, le choix des photographies constitutives du corpus, etc. La Mission du paysage propose un appui technique à l'acteur opérationnel qu'est le partenaire local. À l'échelle nationale, il assure un encadrement par ses actions d'archivage, de diffusion, d'analyse et de valorisation: des représentations et données produites, mais aussi de la méthode de l'observatoire elle-même, qui se précise et se complexifie au fil des années, devenant le principal sujet de questionnement du programme. 


\section{L'art et la méthode, l'art de la méthode : ambivalences}

19 L'idée innovante, servie en principe par l'infrastructure méthodologique, est celle de faire appel à des artistes-photographes, comme en témoigne la première note d'intention du chef de la Mission du paysage. Y sont évoqués, entre autres, des relevés photographiques "effectués par des photographes de haut niveau sur un ensemble d'itinéraires représentatifs de milieux naturels, agricoles, périurbains", pour lesquels «les photographes auraient carte blanche pour saisir à travers leur objectif la réalité qu'ils ressentent ${ }^{20}$.» L'OPNP puise, en effet, son origine dans une reconnaissance de l'intelligence du regard du photographe, reconnu apte à articuler une compréhension sensible et technique des données immatérielles et matérielles qui font le paysage. Ainsi, évoquant l'expérience des premiers itinéraires (initiés alors que la méthode était encore en voie de conception), Caroline Mollie-Stefulesco constate que :

«Confrontés aux réalités du terrain, engagés dans un dialogue avec les aménageurs, ces artistes faisaient la preuve de la qualité de leur intuition, de leur capacité à traquer dans le paysage les signes révélateurs des valeurs d'une société, de ses aspirations, de ses dérives. Leurs images font office de miroir en renvoyant au regard ce qu'il évite souvent de voir ${ }^{21}$. »

"Les photographes sont des chercheurs ${ }^{22}$ ", soutient Jean Cabanel, et l'enjeu premier de la méthode est donc la mise en œuvre du postulat original de la pratique artistique comme forme possible de compréhension du monde. Il apparait pourtant qu'au fil du temps la dimension méthodologique et formelle prend le pas sur cette tentative de production intialement technico-artistique de la photographie.

21 La méthode de l'Observatoire revêt en réalité plusieurs dimensions et se construit sur un équilibre entre art et technique qui peut produire des effets à rebours de sa raison d'être initiale. Le principal effet inattendu et paradoxal est l'effacement du photographique. Afin de concilier technicité et sensibilité et de garantir un usage des données, la «machine archivistique» surdétermine les contraintes imposées au photographe et déplace l'attention sur les pièces non-photographiques (cahiers des charges, carnets de route, comptes rendus, etc.) qui, bien que faisant partie de chaque itinéraire, détournent l'énergie et le regard des images. De la théorie au terrain, le projet photographique se trouve effacé au profit du projet administratif. Et, aucune des successives réactualisations de la méthode - la dernière date de 2009 - ne propose d'éléments pour l'exploitation des images comme outils d'observation des évolutions territoriales ${ }^{23}$. De plus, depuis 1999 , le programme est mis en veille ${ }^{24}$ et la Mission, devenue entre-temps le Bureau des Paysages, n'assure plus d'encadrement à l'échelle nationale. Cet encadrement était non seulement technique et méthodologique, mais aussi culturel dans la mesure où il accompagnait les acteurs locaux dans l'acquisition d'une culture visuelle et photographique qui leur permettait, dans un premier, temps de comprendre les contraintes imposées par la méthode (l'appel à un photographe-artiste, les règles de la re-photographie, etc.) et d'envisager, ensuite, les usages possibles des images comme objets à la fois techniques et culturels.

Ce strict cadre méthodologique et administratif, qui rend l'archive de l'observatoire ${ }^{25}$ finalement peu visuelle, montre que le programme est avant tout un dialogue entre des acteurs réunis autour d'un projet de territoire. C'est du débat, d'avantage que de l'observation des photographies, que découlent les décisions. Préalablement au débat, la 
conception et la mise en œuvre de chaque itinéraire repose sur un "faire ensemble " conceptualisé dans ses directives par la Mission du paysage. Manifestes d'un idéal de projet collectif, celles-ci mettent en correspondance la production de représentations et la production du paysage in situ et s'affilient avec l'idée du paysage portée par l'institution et les champs spécialisés : comme projet collectif, comme bien commun, comme vecteur démocratique. Si l'infrastructure méthodologique semble dans certains cas gommer les particularités de l'artiste-photographe et le dessaisir de sa position d'auteur (le photographe est sollicité pour son regard), elle donne néanmoins à réfléchir sur l'effet de « co-auctorialité » produit par l'observatoire ${ }^{26}$.

\section{Représentativité, comparaisons et « mise en conformité »}

La méthode administrative et photographique de l'observatoire est conçue comme une infrastructure de mise en réseau et de comparaison des représentations, paysages et évolutions territoriales, à l'échelle à la fois locale et nationale. Outre l'ambition d'observation des "temps des territoires", il s'agit de révéler une certaine identité française incarnée dans les paysages. C'est la raison pour laquelle les premiers itinéraires sont conçus sur des territoires ruraux, avec le Parc naturel régional du Pilat, sur des espaces connaissant des explosions périurbaines, comme dans le département de l'Hérault, sur des sites de construction de grandes infrastructures de transports, comme dans le Nord-Pas-de-Calais : autant de territoires illustrant à la fois la diversité des paysages français et la variété des évolutions à l'œuvre. La forme photographique homogène, induite par la méthode, produit un corpus équilibré permettant des comparaisons quantitative et qualitative : des images et des réalités physiques. Ce corpus, qui contribue à actualiser une culture du paysage et de la représentation, parce qu'il est le produit d'une pensée plus générale et d'une sorte de "réglementation photographique ", fait émerger un genre nouveau : la « photographie d'observatoire ${ }^{27} »$ (ill. 5-7). 
III. 5 : Condrieu (69), D 28, vue sur le Rhône et abords (01000101), octobre 1992

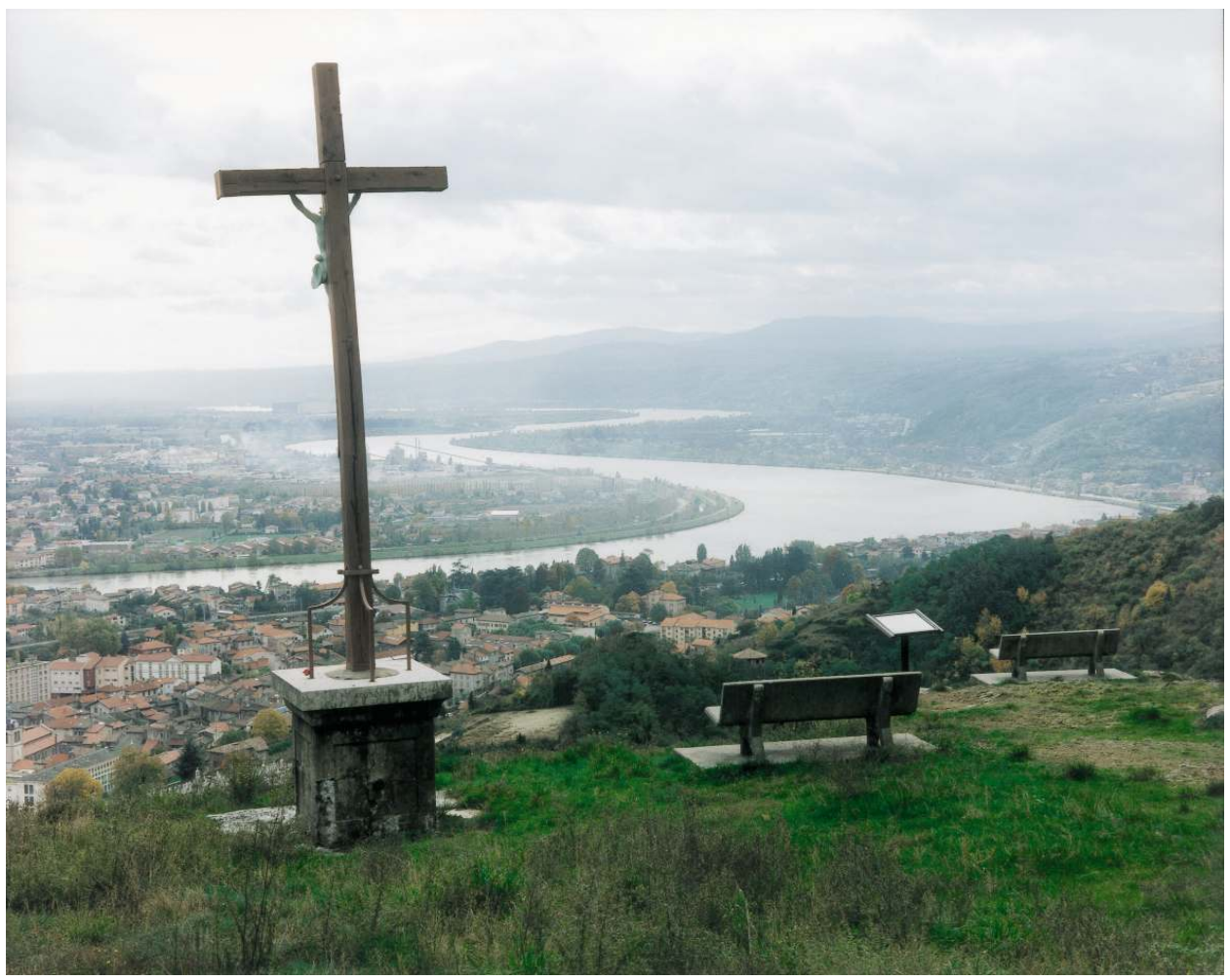

Itinéraire n 1, Parc naturel régional du Pilat, créé en 1990.

(c) Cl. Sophie Ristelhueber, Observatoire photographique national du paysage. Photographie originale en couleurs 
III. 6 : Noyelles-Godault, zone d'activité hôtelière, l'A1 et le terril 28 en arrière-plan (004000201), juin 1993

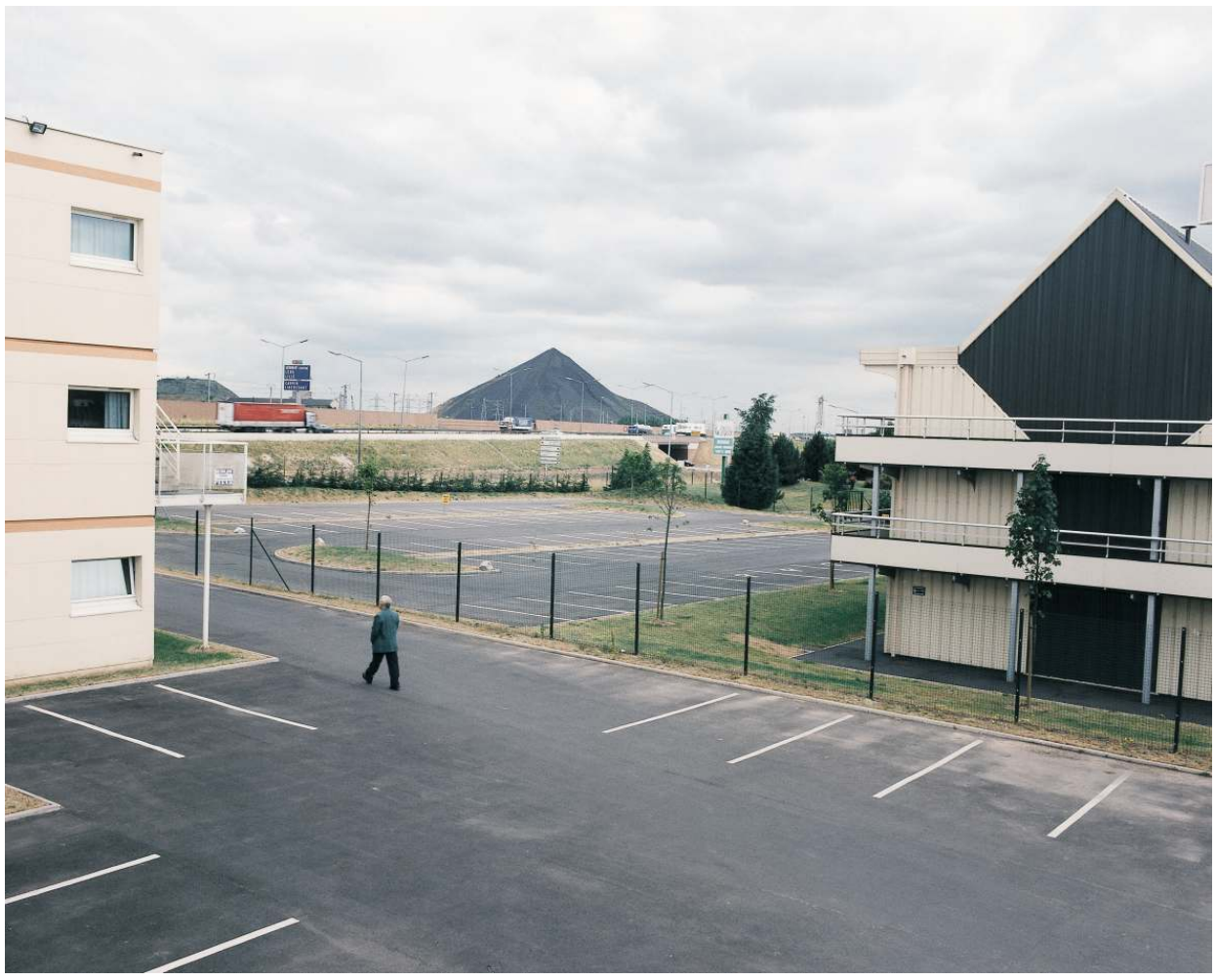

Itinéraire n4, Nord-Pas-de-Calais (DREAL), créé en 1993.

(C) Cl. Dominique Auerbacher, Observatoire photographique national du paysage. Photographie originale en couleurs. 


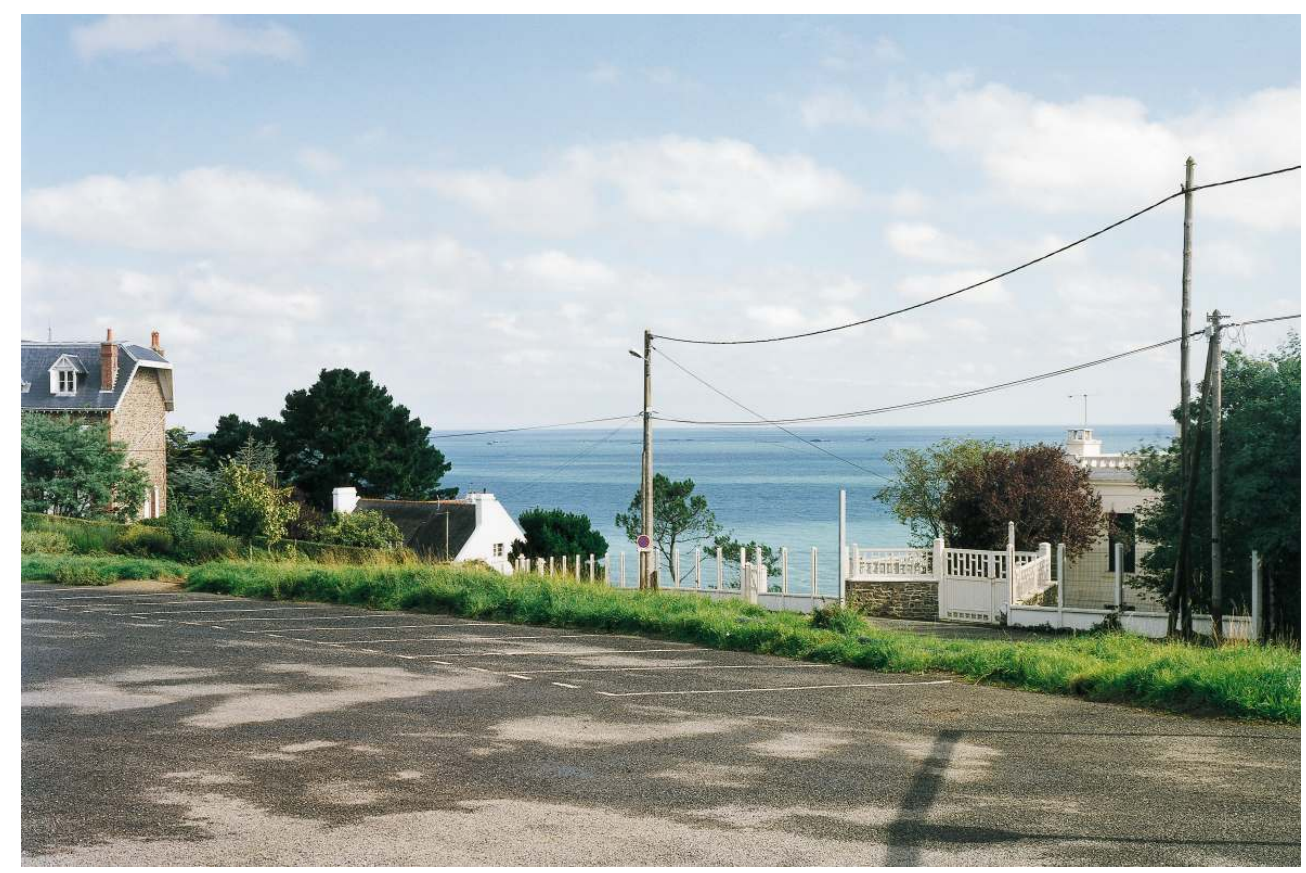

Itinéraire n 5, Côtes d'Armor (CAUE), créé en 1994.

(c) Cl. Thibaut Cuisset, Observatoire photographique national du paysage. Photographie originale en couleurs

Finalement, une nouvelle problématique émerge, qui dépasse l'objet photographique qu'est l'Observatoire : tout genre implique un certain académisme et contient les règles et moyens d'appréciation d'une certaine conformité ou non-conformité. En l'occurrence, cette " photographie d'Observatoire » hérite, produit et transmet une idée de l'aspiration paysagère des commanditaires locaux et nationaux. Elle combine, par son mode même de production, une dimension prescriptive avec des fonctions essentiellement constative et descriptive de la photographie.

En expliquant qu'il s'agit "d'orienter favorablement l'évolution des paysages ${ }^{28}$ ", Jean Cabanel exprime qu'il est lui-même porteur, d'une part, d'un idéal paysager et, d'autre part, d'une volonté de «mise en conformité ». Il s'agit de mettre en œuvre cette mise en conformité par la conception d'un outil spécifique. La "photographie d'Observatoire", forte de l'infrastructure qui la fonde et la porte, peut être appréhendée comme une pratique de représentation et, plus encore, comme une pratique préalable d'aménagement et de mise en conformité : des images et d'une culture du paysage, mais aussi, dans un mouvement incessant d'aller-retour, des espaces eux-mêmes. Ces effets de normalisation des représentations, des regards, des états de réel, des cultures et imaginaires des paysages ne sont pas propres à l'observatoire photographique national du paysage mais sont inhérents à toute démarche de fabrication d'image. Dès lors que celle-ci est portée par un corps collectif, et plus encore produite par une institution par le biais de la commande, l'image produit et diffuse une forme idéale et idéologique : non seulement du sujet représenté, mais aussi de la forme de représentation et de la forme archivistique. 


\section{NOTES}

1. Procès-verbaux de la commission des monuments historiques, séance du 9 mai 1851, archives du Patrimoine, 80/15/7.

2. Anne de Mondenard, La Mission héliographique : cinq photographes parcourent la France en 1851, Paris, Monuments, Éd. du patrimoine, 2002, 320 p.

3. Actuel ministère de l'Écologie, du Développement durable et de l'Énergie.

4. Loi n 93-24 du 8 janvier 1993.

5. Adoptée le 20 octobre 2000 à Florence par vingt-neuf États membres, elle entre en vigueur en France le $1^{\text {er }}$ juillet 2006.

6. Alain Roger (dir.), La Théorie du paysage en France (1974-1994), Seyssel, Pays : Paysages, Champ Vallon, 1995, $464 \mathrm{p}$.

7. Convention européenne du paysage, chapitre « dispositions générales », Article 1 : définitions.

8. Délégation interministérielle à l'aménagement du territoire et à l'attractivité régionale.

9. Gaëlle Morel, «Entre art et culture », Études photographiques, n 16, Mai 2005, [En ligne], mis en ligne le 9 septembre 2008. URL : http://etudesphotographiques.revues.org/715.

10. Devient le Bureau des paysages et de la publicité en 1998.

11. Entretien du 25 novembre 2015.

12. Ibid.

13. Paysages, photographies, travaux en cours, 1984-1985, Mission photographique de la Datar, Paris, Hazan, 1985, 517 p. ; Paysages, photographies, 1984-1988, Mission photographique de la Datar, Paris, Hazan, 1989, 683 p.

14. En complément, du moins dans le projet d'origine des Atlas de Paysages, outil publié en 1994.

15. Mark Klett, Second view. The Rephotographic Survey Project, Albuquerque, University of New Mexico Press, 1984, 211 p.

16. Alain Blondel, Laurent Sully-Jaulmes, Un Siècle passe: 39 photos-constats 1910-1970-1990, Paris, Carré, 1994, n. p.

17. Benoît Coutancier, Gérard Brunot, Luce Lebart, Restaurer la montagne. Photographies et eaux et forêts du XIXe siècle, Paris, Somogy éd. d'art, $188 \mathrm{p}$.

18. Pour des descriptions plus complètes, voir Caroline Mollie (dir.), Daniel Quesney, "Observatoire - Mode d'emploi, Séquences Paysages", Revue de l'Observatoire photographique du paysage, Paris, ministère de l'Environnement/ARP, 2000, p. 104-106.

19. L'Observatoire photographique du paysage, brochure d'exposition, Cité des Sciences et de l'Industrie/ministère de l'Environnement, 1994, $36 \mathrm{p}$.

20. Note relative à la constitution d'un Observatoire sur le paysage, 16 mars 1989.

21. Caroline Mollie, "Huit ans d'Observatoire", Séquences Paysages. Revue de l'Observatoire photographique du paysage, Paris, ministère de l'Environnement/ARP, 2000, p. 8.

22. Entretien du 6 janvier 2016.

23. Caroline Mollie-Stefulesco explique qu'était projetée une deuxième phase durant laquelle ce second pan méthodologique devait être précisé, mais que le ralentissement de l'effort (notamment financier) du ministère envers l'Observatoire, à partir de 1999, a empêché sa mise en œuvre. Entretien du 25 novembre 2015.

24. De nombreux observatoires photographiques dits « locaux » se créent néanmoins, produisant un maillage irrégulier sur le territoire et démontrant l'intérêt vif que cet outil suscite auprès des acteurs concernés. 
25. Dans sa version "nationale». On distingue aujourd'hui l'OPNP des OPP, Observatoires photographiques du paysage, dits « locaux».

26. Julia Bonaccorsi et Anne Jarrigeon, «Les Observatoires photographiques ou la production d'un regard public sur le paysage ", Les Médiations documentaires. Nouvelles études anthropologiques, l'Harmattan, Paris, 2014, 208 p.

27. Expression employée parfois péjorativement par les photographes.

28. Conseil des ministres le 22 novembre 1989.

\section{RÉSUMÉS}

L'Observatoire photographique national du paysage, lancé en 1991 par le ministère de l'Environnement, est un programme visant à constituer une archive photographique de la diversité des paysages français. Fondé notamment sur l'objectif d'appréhender les rythmes et temps des territoires, et mu par l'hypothèse de la photographie comme un vecteur de révélation des évolutions des espaces, le programme de l'Observatoire envisage le projet de l'artiste comme moyen de réflexion et comme outil d'action. Ces postulats originaux connaissent aujourd'hui un intérêt renouvelé de la part des acteurs des territoires et de l'aménagement. Ainsi, la méthode de l'observatoire est devenue un modèle pour la création d'une dynamique de représentation photographique des paysages sur l'ensemble du territoire français. Cet article revient sur les origines historiques, les fondements conceptuels, les enjeux et les objectifs, à la fois théoriques, politiques, artistiques et techniques, de cette machine archivistique qui allie rétrospectif et prospectif. Plus largement, il s'agit d'esquisser, par l'analyse de ce programme institutionnel, des pistes de réflexion sur les processus de fabrication de nos manières de voir, de représenter et de projeter les paysages.

The Ministry of the Environment launched in 1991 the National photographic landscape observatory. This program, based on the hypothesis that the photographic medium is able to reveal spatial evolutions in a specific way, aims to create a representative archive of the landscapes diversities and evolutions. In a new and specific way, the administration puts the artistic dimension at the center of a technical and operational tool. Today, with a new dynamic, the local institutions use the Observatory method as a model to document their territories and their actions and create a national movement of landscape representation. This article aims to explain the origins, the historic and conceptual foundations along with the theoretical, political, artistic and technical goals of this recording machine, which has simultaneously a retrospective and a prospective dimension. And the analysis of this institutional program is a way to outline a reflexion about the fabrication process of our way of seeing, representing and conceiving everyday landscapes.

Das im Jahr 1991 vom französischen Umweltministerium begründete Observatoire photographique national du paysage ist von der Zielsetzung geleitet, ein fotografisches Archiv der Vielfalt der französischen Landschaften zu erstellen. Mit dem Ziel, den Rhythmus und die Zeitwahrnehmung in den einzelnen Landstrichen zu erfassen, sowie der Auffassung, dass räumliche Entwicklung sich insbesondere über das Medium der Fotografie offenbare, setzt das Observatoire künstlerische Projekte sowohl als Reflexionsmittel als auch als Handlungsinstrument ein. Heute kommt der ursprünglichen Zielsetzung des Projekts von Seiten der Stadt- und Regionalplanung wieder eine 
gesteigerte Aufmerksamkeit zu. So ist das methodische Vorgehen des Observatoire ein Modell für die Erstellung von fotografischen Landschaftsaufnahmen im gesamten französischen Staatsgebiet geworden. Dieser Beitrag beschäftigt sich mit den historischen Hintergründen, den konzeptuellen Grundlagen und den theoretischen, politischen, künstlerischen und technischen Zielsetzungen dieses archivarischen Projektes, das eine retrospektive wie prospektive Dimension vereint. Darüber hinaus handelt es sich darum, mit der Untersuchung dieses institutionellen Programms weitere Forschungsfragen aufzuzeigen, die sich mit der Konstituierung unserer Sichtweise sowie unserer Darstellungs- und Projektionsart von Landschaften beschäftigen.

\section{AUTEUR}

\section{FRÉDÉRIQUE MOCQUET}

Frédérique Mocquet est née en 1986. Elle est diplômée de l'École nationale supérieure d'arts appliqués et des métiers d'arts Olivier de Serres (2008) et de l'École nationale supérieure d'architecture Paris-Malaquais (2012). Depuis 2013, elle poursuit ses recherches dans le cadre d'un doctorat en architecture (laboratoire Infrastructure-Architecture-Territoire, École doctorale Ville-Transport-Territoire) sous la direction de Jacques Fol. Son travail, intitulé Paysages photographiques : représentations, aménagement et prospective, analyse des dispositifs en tension entre enjeux documentaires et ambitions créatives et s'interroge sur les possibilités des pratiques photographiques comme moyens et outils d'expériences et de connaissance des évolutions spatiales. Adresse électronique : f.mocquet@gmail.com 